\title{
Non-Invasive Measurement of Internal Temperature of a Cylindrical Li-ion Cell During High-Rate Discharge
}

\author{
Dean Anthony ${ }^{1}$, Derek Wong ${ }^{2}$, David Wetz ${ }^{2}$, Ankur Jain ${ }^{1, *}$ \\ ${ }^{1}$ - Mechanical and Aerospace Engineering Department \\ 2 - Electrical Engineering Department \\ University of Texas at Arlington, Arlington, TX, USA. \\ * Corresponding Author: email: jaina@uta.edu; \\ 500 W First St, Rm 211, Arlington, TX, USA 76019 \\ Ph: +1 (817) 272-9338; Fax: +1 (817) 2722952
}

\section{Abstract}

Li-ion cells are a technologically important class of devices for electrochemical energy storage and conversion. Overheating of a $\mathrm{Li}$-ion cell during operation is undesirable as it directly affects performance and safety. Although a number of methods have been used for temperature measurement in Li-ion cells, there is a lack of non-invasive techniques to determine the peak temperature at the core of the cell. Measuring only the outside surface temperature, while straightforward, is not sufficient since the core temperature is in most cases much higher. This paper presents non-invasive measurement of the core temperature of a $\mathrm{Li}$-ion cell using a recently developed technique that utilizes space and time integrals of the measured temperature field at the outside surface. The surface temperature field of an operating Li-ion cell is measured using infrared thermography at multiple discharge rates up to $10 \mathrm{C}$, using which, the core temperature is predicted as a function of time. In each case, there is excellent agreement throughout the discharge period between the predicted core temperature and measurements from a thermocouple embedded at the core of the cell. These measurements quantify the temperature gradient within the cell, which is particularly high at large discharge rates. This paper may result 
in non-invasive core measurement methods that may contribute towards performance optimization and improved safety of Li-ion cells.

Keywords: Lithium Ion Battery; Temperature Measurement; Battery Safety; Thermal Management.

\section{Introduction}

Accurate measurement of temperature in a Li-ion cell is a critical research challenge for ensuring performance, safety and reliability of systems that utilize Li-ion cells for energy conversion and storage [1-3]. Temperature rise occurs in a Li-ion cell due to heat generation during energy conversion, particularly at high discharge rates [4-6]. The amount of heat generated in a typical cylindrical $26650 \mathrm{Li}$-ion cell is not extremely large. For example, about 10W heat generation has been reported during discharge at an aggressive 10C rate [7,8]. For reference, the C-rate of a charge/discharge process is defined as the reciprocal of the number of hours needed to complete charge or discharge the cell [1,7]. However, due to poor thermal conductance of the cell [9] resulting from material and interfacial thermal resistances in the cell [10], this causes significant temperature rise [11,12], particularly in the core of the cell, where heat accumulation tends to occur due to the lack of a direct access for heat removal [13]. While high core temperature may improve cell performance due to reduced internal resistance, it is also known that high cell temperature increases the rate of capacity fade $[14,15]$. Further, in many cases, the Battery Management System (BMS) may throttle cell power in response to a high cell temperature to keep the cell within a safe envelope, thereby resulting in reduced performance . This necessitates the design and implementation of an effective thermal management strategy in order to keep the cell temperature below a threshold value and prevent undesirable conditions such as thermal runaway [16-18]. A number of thermal management strategies have been 
reported in the past, which have been well covered by recent review articles $[14,15,19]$. Most thermal management techniques focus on heat removal from the outside surface of the cell. This is accomplished either by the flow of an appropriate coolant such as air or water over the cell surface, or by cold plates [20], heat pipes [21] or phase change materials [22] in the battery pack. Other more intrusive thermal management techniques include the use of electrolyte as a coolant [23], heat absorption from current collector foils [24], as well as the integration of a heat pipe protruding out of the cell [13].

Accurate measurement of temperature of the cell, particularly the peak temperature that occurs at the core of the cell is critical for effective thermal management and thermal control. Measurement of surface temperature alone does not provide much information about the core temperature since the surface temperature may not rise much even when the core is much hotter and is in urgent need for cooling. In past experiments, for example, a temperature difference of as much as $15{ }^{\circ} \mathrm{C}$ has been reported between surface and core temperatures of a Li-ion cell undergoing high rate discharge [7,25]. Therefore, thermal management based on surface temperature measurement may lead to insufficient cooling, and measurement of the core temperature is critical. This may improve not only the effectiveness of thermal management but also electrochemical performance optimization of Li-ion batteries. For example, core temperature measurement of multiple cells in a battery pack may be used for load balancing within the pack by decreasing the load on an overheated cell.

Due to its importance in thermal control, a significant effort has been expended in several other engineering systems to measure the core temperature. For example, in computer microprocessors, temperature sensors are embedded deep within the microprocessor chip where 
the highest temperature is expected to be encountered [26]. Similar core temperature measurement is also desirable for Li-ion cells. While the temperature on the surface of a Li-ion cell can be easily measured using thermocouples or other thermal sensors [7], measurement of the core temperature is not straight-forward. The electrochemically active environment inside a Li-ion cell presents challenges for the survival of any temperature sensor embedded within. Due to the hermetically sealed nature of the cell, connecting to an embedded sensor is also difficult. Thermocouples have been inserted in Li-ion cells $[7,8,25,27]$, but this approach is unreliable and often causes cell failure. Some research has been carried out to utilize certain electrochemical characteristics of the cell as an indicator of its temperature [28], but this approach only yields the volume-averaged temperature, and does not give information about the peak temperature at the core of the cell. The insertion of fiber Bragg sensors has also been investigated [29], however, this approach is expensive and cumbersome, and unlikely to be a candidate for implementation at a large scale. Non-invasive temperature measurement techniques such as ultrasonic temperature measurement - in which the speed of propagation of an ultrasonic wave through a device is used to determine the average temperature along the path [30] - have not been used for a Li-ion cell, possibly due to lack of data on speed of sound as a function of temperature in a Li-ion cell. Given the shortcomings of the current state-of-the-art, development of new, non-intrusive core temperature measurement techniques is very desirable for Li-ion cells. This is likely to have a direct impact not only on thermal management of the cell, but also on real-time performance optimization.

In the recent past, a transduction-free method for measurement of core temperature in a heat-generating cylinder based on measurement of surface temperature fields has been developed [31,32]. In this method, the steady-state [31] or transient [32] core temperature of the cylinder 
has been shown to be related to surface temperatures through appropriate space and time integrals. Accurate measurement of the core temperature based on non-invasive, infra-red (IR) measurement of the surface temperature field has been demonstrated. This method has the advantage of being non-intrusive and based entirely on the surface temperature, which is relatively easy to measure. Past papers [31,32] have demonstrated this method for nonelectrochemical test cells in which heat is generated through Joule heating, a much simpler mechanism compared to active electrochemistry inside a Li-ion cell.

This paper presents non-invasive measurement of the core temperature of a $26650 \mathrm{Li}$-ion cell during high-rate discharge. The core temperature is determined using a theoretical model that utilizes measurements of the transient temperature field on the outside surface of the cell. Core temperature is measured in this manner for multiple discharge rates, and for a discharge process involving sudden change in discharge rate. In each case, good agreement is found with independent measurements of the core temperature using an embedded thermocouple. These experiments demonstrate an effective, non-invasive method for determining the core temperature of an operating Li-ion cell, which may be helpful for thermal control, performance optimization as well as monitoring of thermal runaway.

\section{Theoretical Model}

A theoretical model to predict the core temperature of a heat-generating cylinder as a function of time based on measurements of the temperature field on the outside surface of the cylinder has been presented in a recent paper [32]. Figure 1(a) shows a schematic of an infinite cylinder of radius $R$ with volumetric heat generation $Q(t)$ occurring within. This model can be applied to a heat-generating, cylindrical Li-ion cell shown in Figure 1(b), for which the core 
temperature $T_{0}(t)$ is of interest. The outside surface temperature of the cylinder, $T_{\text {out }}(\theta, t)$ is measured circumferentially and as a function of time. When the heat generation rate $Q(t)$ is known, it has been shown [32] that utilizing the measured outside temperature as a transient boundary condition, the governing energy conservation equations can be solved using the method of undetermined parameters. This has been shown [32] to result in the following expression for the core temperature $T_{0}(t)$

$$
T_{0}(t)=T_{0, A}(t)+T_{0, B}(t)=\left[\frac{Q R^{2}}{4 k_{r}}+\sum_{n=1}^{\infty} u_{n} e^{-\alpha_{r} \mu_{n}^{2} \cdot t}\right]+\sum_{n=1}^{\infty} v_{0 n}(t)
$$

where $k_{r}$ and $\alpha_{r}$ are the thermal conductivity and thermal diffusivity respectively of the cylinder material. The coefficients $u_{n}$ and $v_{o_{n}}(t)$ are given by

$$
u_{n}=\frac{-\frac{Q}{4 k_{r}} \int_{0}^{R}\left(R^{2}-r^{2}\right) \cdot r J_{0}\left(\mu_{n} r\right) d r}{N_{r, n}}
$$

and,

$$
v_{0 n}(t)=\frac{\alpha_{r} \mu_{n} R J_{1}\left(\mu_{n} R\right)}{N_{r, n}} \int_{0}^{t} w_{0 I}(\tau) \exp \left[-\alpha_{r} \mu_{n}^{2}(t-\tau)\right] d \tau
$$

In equations (2)-(3), $\mu_{n} R$ are roots of $J_{0}$, the Bessel function of the first kind and order zero. The eigenfunction normalization integral $N_{r, n}$ is given by

$$
N_{r, n}=\frac{R^{2} J_{1}\left(\mu_{n} R\right)^{2}}{2}
$$

Finally, $w_{0 I}(\tau)$ is the circumferential integral of the outside temperature $T_{\text {out }}(\theta, \tau)$ at any time, 
given by

$$
w_{0 I}(\tau)=\frac{1}{2 \pi} \int_{0}^{2 \pi} T_{\text {out }}(\theta, \tau) d \theta
$$

Note that when the heat generation rate $Q$ is a function of time, the first term $T_{0, A}(t)$ in equation (1) must be replaced by

$$
T_{0, A}(t)=L^{-1}\left[\frac{\alpha_{r} \bar{Q}}{s k_{r}}\left(1-\frac{1}{I_{0}\left(\sqrt{\frac{s}{\alpha_{r}} R}\right)}\right)\right]
$$

where $L^{-1}$ is the inverse Laplace transform, $\bar{Q}$ is the Laplace transform of $Q(t)$, and $I_{0}$ refers to modified Bessel function of the first kind and of order zero. The Laplace transform $\bar{Q}$ can be determined once the expression for $\mathrm{Q}(\mathrm{t})$ is known, which may vary either smoothly or sharply with time.

Equation (1) provides the basis for determining the core temperature of a $\mathrm{Li}$-ion cell undergoing a discharge process that generates a certain amount of heat based on measurements of the outside surface temperature. Note that the core temperature at any given time depends on the measured surface temperature field at all prior times, which must be integrated in space as shown in equation (5) and then in time as shown in equation (3). Details of this key theoretical result may be obtained from a recent paper [32].

\section{Experimental Methods}

\subsection{Assembly of Li-ion cell with embedded core thermocouple}

In order to fabricate an operating cell with a core-embedded thermocouple, Lithium iron 
phosphate (LFP) cells of a 26650 form factor are received from the manufacturer, as shown in Figure 2(a). The cells have no electrolyte and are unsealed, which allows for thermocouple insertion before the cell becomes electrochemically active. To precisely mimic the performance of production cells, electrolyte of the same formulation used in the production cells (1:1:1 of EC:DEC:DMC with $\mathrm{LiPF}_{6}$ salt) is obtained. The production version of these cells has nominal capacity of $2.85 \mathrm{Ah}$, and is capable of operating between a maximum range of $2.0 \mathrm{~V}$ to $4.1 \mathrm{~V}$ at a maximum rate of 42 A continuous discharge and 5 A continuous recharge. The nominal voltage for these cells is $3.2 \mathrm{~V}$ during discharge at the nominal $1 \mathrm{C}$ rate of $2.85 \mathrm{~A}$. In order to ensure the best seal possible for the cell after fabrication, a hole is first drilled in the underside pressure cap below the top nip connection on the positive terminal, as shown in Figure 2(b). During drilling, the cell is mounted on its side and the opening exposing the jelly roll is covered to prevent any metal shavings from falling in. After this is accomplished, a $2.3 \mathrm{~mm}$ steel rod is used to widen the space in the center of the jelly roll in order to create enough space for the thermocouple assembly to be inserted into the core.

The thermocouple assembly consists of three thermocouples, which are epoxied to the inside of a heat shrink tube at different lengths from the base of the cell, as shown in Figure 2(c). When the epoxy is dried out, a heat gun is used to collapse the heat shrink around the thermocouples. This assembly is then carefully inserted into the core of the test cell and the other end of the thermocouple wires are fed through the drilled hole and out of the small ports between the top nip and the pressure cap, as shown in Figure 2(d). The hole in the pressure cap is then filled with marine epoxy, and the cell is mounted upside down to allow the resin to fill the entire cavity between the nip and the pressure cap. This ensures a robust seal to avoid leakage of electrolyte and reactivity with the ambient environment. The cell is then left for twenty-four 
hours to allow time for the epoxy to cure completely before electrolyte filling begins.

After the marine epoxy completely cures, the cell is transferred into a glove box and filled with $10 \mathrm{~mL}$ electrolyte solution using a $3 \mathrm{~mL}$ long needle syringe, as shown in Figure 2(e). The electrolyte is introduced in stages to ensure complete filling. Three to five filling stages are required to completely introduce all $10 \mathrm{~mL}$ into the cell. In each of these stages, electrolyte is introduced until the level is near the brim of the casing. Fifteen minutes are allowed between stages or until the level of electrolyte solution had gone down far enough for another session to begin. This ensures that the electrode films are saturated with electrolyte solution. After this is complete, the cell is ready to be crimped.

In order to press the crimping dies for these cells within the glove box, a makeshift hydraulic press is constructed by using two steel plates and a hydraulic lifting cell as shown in Figure 2(f). The first die bends the top lip into a cone shape, while the next two dies are flat, which press the bent lip firmly against the rubber seal. The dies have a through hole in the center to allow the thermocouple wires to be fed through and prevent damage from compression. After the three stages of dies have been pressed onto the cell, it is allowed to sit in the glove box for twenty-four hours to achieve chemical neutrality.

\subsection{Experimental setup for core temperature measurement}

A picture of the experimental setup and a schematic of the experimental data flow for non-invasively measuring the core temperature of the fabricated Li-ion cell using surface temperature measurements, are shown in Figure 3. The Li-ion cell is placed on two thin, tapered foam risers to minimize stray heat loss, and placed on an optical breadboard directly under a FLIR A6703 InSb infrared camera. The external surface of the Li-ion cell is covered with a DGF graphite aerosol spray to ensure a high and uniform surface emissivity. As shown in Figure 3(a), 
lead wires from a MACCOR Series 4000 Automated Test System are attached to the power leads of the cell for charging and discharging the cell. A separate set of wires also connect the cell to a PARSTAT 4000 Potentiostat for EIS testing between experiments. The cell is subjected to a variety of programmable charging and discharging profiles. A small Fugetek HT-07530D12 computer fan is used for forced convection experiments to provide cooling to the Li-ion cell. The fan is placed on a metal riser in order to align the fan with the longitudinal axis of the Li-ion cell. This ensures direct and symmetrical impingement of air from the fan to the cell. A variable resistor controller is then used to control the speed of the fan for variable fan speed experiments. Air speed from the fan is measured using an Extech mini thermo-anemometer to be around 3.0 $\mathrm{m} / \mathrm{s}$. Thermocouples embedded in the cell are connected to a NI-9213 DAQ thermocouple module. Data acquisition is carried out through LabView software with a frequency of $1 \mathrm{~Hz}$. Infrared temperature measurements are carried out using ResearchIR software, also at a frequency of $1 \mathrm{~Hz}$. Both measurements are carried out once every second. This measurement rate is appropriate for this system which has a relatively larger time constant (estimated to be around 48 seconds based on forced air cooling of a 26650 cell) compared to the data acquisition time interval.

\subsection{Electrochemical measurements}

The initial cycling procedure conducted on the test cell is intended to grow the initial layers of surface film on the electrodes. The cell is charged at $0.56 \mathrm{~A}$ to $4.1 \mathrm{~V}$ using a constant current-constant voltage profile $(\mathrm{CC}-\mathrm{CV})$ and a current cutoff of $0.30 \mathrm{~A}$. While the maximum voltage for this chemistry is normally $3.65 \mathrm{~V}$, the range is extended in order to promote the formation of the solid electrolyte interphase layer. This surface film is critical to the cycle life of the cell. After this step, the cell is cycled five times at the same rate using the same CC-CV 
procedure and a $\mathrm{CC}$ discharge procedure also at $0.56 \mathrm{~A}$. To ensure that the cell is safe to operate, an initial discharge is conducted at approximately ten times the nominal rate (25 A).

After the high rate discharge test, the cell is recharged to $100 \%$ state of charge (SOC). An initial galvanic EIS spectrum is collected from $1 \mathrm{kHz}$ to $5 \mathrm{mHz}$ with a $1.00 \mathrm{~A}$ amplitude. For comparison, EIS of a baseline cell filled and sealed without any thermocouples inserted in its core is also carried out. The EIS spectra presented in Figure 4 show very similar electrochemical behavior when comparing the control cell (dashed line) with the thermocouple cell (solid line). There is only a slight increase in the real impedance of the cell due to insertion of the thermocouple assembly. The magnitude of the imaginary impedance in the mid-frequency semicircle for the thermocouple cell is only $1 \mathrm{~m} \Omega$ less than that of the control cell. This indicates that while the Ohmic resistance of the cell is increased by the presence of the thermocouple assembly, there is no reaction with battery chemicals and active material. The Ohmic resistance, which is found from the $\mathrm{x}$-axis intercept of the Nyquist plot, is only a few $\mathrm{m} \Omega$ greater than that of the baseline cell. Similarity between the test and control cells is further supported by the observation that the Warburg tail in the low frequency region after the initial semi-circle remains virtually unchanged. The slope of the Warburg tail is nearly the same for the control cell and the test cell, which shows that impedance to diffusional processes such as the transport of Lithium-ions in electrode materials remains unaffected by the presence of the thermocouple assembly. Thus, in comparison to the control cell, the test cell does not exhibit any abrupt change in its electrochemical behavior due to the presence of the embedded thermocouple.

\subsection{Core temperature measurements}

Infrared temperature measurement usually requires exhaustive calibration. In this case, the surface of interest is coated with a graphite film, which is calibrated in advance by measuring 
the temperature of an Instec HCS662V thermal stage coated with the same graphite spray. Since the same graphite spray is used in all experiments, the calibration needs to be performed only once. Measurements of the surface temperature during a defined temperature ramp are compared with the true temperature of the stage measured by an embedded thermocouple, showing very close agreement between the two for the entire ramp duration, with a peak deviation of only 0.31 ${ }^{\circ} \mathrm{C}$. This establishes a baseline calibration for the camera and shows the capability of accurately measuring the temperature of a surface coated with graphite spray.

Once calibration is completed, a number of experiments are carried out at different discharge rates in order to measure the core temperature of the Li-ion cell using the method outlined in section 2. In each case, the Li-ion cell starts fully charged at ambient temperature, and a constant or time-varying discharge is conducted using the MACCOR cycler. The temperature distribution on the surface of the cell is measured in time using the infrared camera while the cell potential reaches $2.0 \mathrm{~V}$. This is controlled by a shut off flag in the cycling procedure. The temperature field around the circumference of the cell at mid-height is extracted from the measured two-dimensional temperature field from the infrared camera. These data are subjected to a mathematical transformation to account for the projected view of the curved surface of the Li-ion cell as viewed by the infrared camera [32].

Three sets of experiments are carried out on the Li-ion cell. First, the core temperature of the cell is measured using surface temperature measurements at a number of discharge rates while the Li-ion cell is cooled via natural convection conditions. Then a set of experiments is carried out for the same discharge rates, with forced convective cooling from a fan operating at various speeds. Finally, experiments are carried out to simulate an anomalous increase in heat generation, as in the case of thermal runaway [18], for each of the previously discussed cooling 
conditions. In these experiments, the MACCOR provides a constant $4 \mathrm{C}$ discharge load for a period of 7.5 minutes, followed by a much larger 10C load for the remainder of the cell capacity, for another 3 minutes.

A stabilization time of a few minutes is provided in each experiment with air flow from the fan before the experiment commences. At the conclusion of each experiment, the cell is charged at a rate of $1 \mathrm{C}$ and allowed to cool for a few hours. An EIS test is carried out to ensure that the EIS profile and cell internal resistance have not changed appreciably before proceeding to the next experiment. An extended cool-down period is provided between experiments to ensure that each experiment begins with the entire cell at ambient temperature. In each experiment, the core temperature of the cell is determined using the experimentally measured surface temperature distribution in conjunction with the theoretical model presented in section 2. These core temperature measurements are compared against measurements from the embedded thermocouple in the $\mathrm{Li}$-ion cell to validate the temperature measurement method.

\section{Results and discussion}

Figure 5 shows results from an experiment in which the 26650 cell with embedded thermocouple is subjected to $10 \mathrm{C}$ discharge while its surface temperature distribution is measured using infrared thermography. Using the surface temperature data and equations (1)-(5), the two components of the core temperature, $T_{0, A}$ and $T_{0, B}$ are calculated as functions of time. 415 eigenvalues are used for $T_{0, A}$ and $T_{0, B}$. Past work suggests slow convergence of the infinite series for $T_{0, B}$ [32], which necessitates computation of a large number of terms. Figure 5 plots these components as well as their sum, which, according to the theoretical model determines the core temperature of the cell as a function of time. For reference, the true core temperature of the cell, 
measured through the embedded thermocouple is also plotted in Figure 5. Figure 5 shows that both $T_{0, A}$ and $T_{0, B}$ increase as the discharge process progresses. $T_{0, A}$ is convex in nature, rising fast initially and then plateauing out. On the other hand, $T_{0, B}$ remains flat to begin with, and then starts rising in a concave fashion. The sum of $T_{0, A}$ and $T_{0, B}$ is found to closely match the actual core temperature of the cell, measured by the embedded thermocouple. The worst-case disagreement between the two over the entire experimental duration is less than $1{ }^{\circ} \mathrm{C}$. This demonstrates the capability of this experimental method to accurately determine the core temperature based on measurements of the surface temperature distribution.

Figure 5 shows that the component $T_{0, B}$ remains relatively small for the first several minutes of the experiment. In such a case, the computational effort in determining the core temperature can be significantly reduced by computing only the $T_{0, A}$ component. If so, it will be important to determine when $T_{0, B}$ begins to become significant, which will depend on the relative values of internal heat generation rate and surface temperature measurement, as well as on the level of accuracy desired from the core temperature measurement.

Note that determining the core temperature in this fashion requires information about the heat generation rate in the cell, as well as various thermal properties of the cell, including thermal conductivity and specific heat. In this case, the heat generation rate is obtained from prior measurements on a 26650 cell [7] that showed that heat generation rate in a 26650 cell increases quadratically with C-rate. Further, since heat generation also depends linearly on the internal resistance of the cell, the heat generation from past measurements is scaled based on the ratio of the internal resistances from EIS measurements. Heat generation rate could also be determined through its dependence on various components of heat generation on electrochemical parameters such as a current, voltage and overpotential. The heat generation rate is assumed to 
be a constant, although in reality, there may be minor variations with time, particularly towards the start and finish of the discharge process [33], which may be the reason behind the increased disagreement between these measurements and thermocouple measurement of the core temperature at the start and finish of the discharge process. Further, there may be spatial nonuniformity in heat generation due to additional Ohmic heat generation at the current tabs inside the cell, although this effect is expected to be negligible for a well designed cell.

Further, note that the thermal conductivity and heat capacity of the 26650 cell are obtained from past papers that have reported measurements of these thermophysical properties [9,34] for a 26650 Li-ion cell.

These experiments are repeated at a number of discharge rates. In each case, the computed core temperature based on the theoretical model is compared against core temperature measurement using the embedded thermocouple during the entire discharge period. This comparison is shown in Figure 6. As expected, the temperature rise is greater for higher C-rates due to increased heat generation rate. There is good agreement between the core temperature prediction and the thermocouple measurement over the entire discharge duration for each C-rate. The agreement between the two is particularly good at higher discharge rates, which is also where accurate measurement of the core temperature is particularly critical due to large temperature rise and the need for effective thermal management. There is somewhat greater disagreement at lower C-rates, possibly because at lower C-rates, the temperature rise itself is so small that measurement uncertainties are relatively larger. Further, variation in heat generation rate as a function of time appears to be more significant at lower C-rates. Regardless, the disagreement between the core temperature determined through surface temperature 
measurements and direct thermocouple measurements is less than $1.4{ }^{\circ} \mathrm{C}$ for the worst-case $1 \mathrm{C}$ discharge and less than $1.0^{\circ} \mathrm{C}$ for the $10 \mathrm{C}$ discharge.

In addition to constant-rate discharge, processes involving multiple rates of discharge are also common and technologically relevant. A change in the rate of discharge may occur when the power load changes, for example, in an electric vehicle due to acceleration and deceleration. In an extreme case involving abuse of the cell, the discharge rate can also go up dramatically due to short circuit. In each case, as the discharge rate increases, so does the rate of heat generation, and hence it becomes critical to be able to monitor the core temperature of the cell during such processes. In order to demonstrate the capability of this core temperature measurement method in such a scenario, the $26650 \mathrm{Li}$-ion cell is discharged, starting at 4C, and then increasing abruptly to 10C. The core temperature of this cell, measured using the embedded thermocouple is compared with the prediction from the theoretical model using surface temperature distribution measurements. There is good agreement between the two, as shown in Figure 7, which shows that the temperature increases slowly during the $4 \mathrm{C}$ discharge, and the rate of temperature rise increases once the discharge rate goes up. During both phases of discharge, the predicted core temperature is in good agreement with measurements from the embedded thermocouple. The somewhat increased discrepancy in this case compared to constant C-rate cases may be due to reduced accuracy in heat generation rate. When the C-rate changes sharply, it is possible that the heat generation rate changes more smoothly. The predicted core temperature is somewhat lower than the thermocouple measurement towards the end of the higher-rate discharge, which could possibly be due to larger-than-expected heat generation rate towards the end of the discharge period. 
A key goal for this method of determining the core temperature of the cell is to provide a better estimate of the thermal health of the cell. In the absence of a method to determine the core temperature of the cell, thermal management and performance optimization may need to be based on surface temperature measurement alone, which is clearly not representative of the peak temperature that must be known for optimal control and thermal management, and which occurs at the core of the cell. Figure 8 plots the non-invasively measured core temperature and the circumferentially averaged surface temperature of the cell as a function of time during a 10C discharge. These data clearly show that while the surface and core temperatures are close to each other at the start of the discharge process, the difference between the two grows as the cell discharges. By the completion of discharge, there is an almost $10{ }^{\circ} \mathrm{C}$ difference between the core and surface temperatures. This demonstrates the importance of using the measured core temperature instead of surface temperature for thermal management and performance optimization. Even with a factor of safety, a surface temperature based thermal design of the cell may fail since the core may be much hotter. Cooling of the cell based on surface temperature will simply not be effective because the surface temperature of the cell is significantly lower than the core temperature, which may lead to under-provisioning of cooling when the core of the cell is actually much hotter and in urgent need of cooling.

Note that as shown in equations (3) and (6), the method described here requires information about the past values of surface temperatures on the cell. While in the present work, the entire past thermal history of the cell was measured through an infrared camera, it would be interesting to determine the extent of reduction in accuracy of the method in predicting the core temperature if incomplete information is available about the past thermal history of the cell. Similarly, while surface temperature measurements have been taken with a very accurate 
infrared camera in this work, there is likely to be reduction in accuracy of the method when there is significant inaccuracy in surface temperature measurement, or when the surface temperature measurement data is available only at discrete points instead of a near-continuous measurement provided by an infrared camera. While this may be acceptable for applications where the accuracy requirement for core temperature measurement is not very high, for other applications, this may present a limitation of this method.

Experimental uncertainty in the measurement of the core temperature arises from uncertainty in the measurement of the underlying parameters used for determining the core temperature. These include thermal conductivity, heat generation rate and surface temperature measurement. The uncertainties in each of these parameters are combined using a standard error propagation analysis [34]. Uncertainties in thermal conductivity and heat generation rate are taken from papers that have reported these measurements [7,9]. Uncertainty in surface temperature measurement is obtained from the calibration curve for the graphite spray to be around $0.31{ }^{\circ} \mathrm{C}$. When combined, the overall uncertainty in the core temperature measurement is estimated to be around $15 \%$. This value may be somewhat larger for lower C-rates, where the temperature rise itself is relatively small. Further, if a less accurate IR camera is used, which offers a greater uncertainty in surface temperature measurement, then the overall uncertainty in core temperature measurement will go up accordingly. When cost is a concern, or when space around the cell is limited, for example in a battery pack in an electric vehicle, the use of multiple thermocouples or other discrete temperature sensors may be needed instead of an IR camera. In such a case, the integral in equation (5) will need to be evaluated approximately using the discrete measurements, and may incur some error that can be minimized by using sufficiently large number of thermocouples and by optimizing the location of the thermocouples. For 
applications such as electric vehicles where direct optical access to a cell may not be possible due to tight packing in a battery pack, these trade-offs must be considered carefully.

\section{Conclusions}

Internal temperature measurement in $\mathrm{Li}$-ion cells is a critical metrology need that directly affects performance and safety. The non-invasive measurement method described in this paper directly addresses this need by determining the core temperature using a theoretical relationship with the outside surface temperature distribution and other parameters predicted by solving the underlying energy conservation equations. The outside temperature distribution is determined through infrared temperature measurements. Core temperature measurements are in close agreement with direct measurements from an embedded thermocouple for a variety of cell discharge conditions.

While measurements have been demonstrated and validated in this paper on cylindrical

cells, a similar approach could be adopted for prismatic cells as well. Further, while the focus of this work is on high rate discharge, a similar approach can be used to determine the core temperature during high rate charging, as long as the heat generation rate is known.

Further work may be needed for utilization of this method for extreme events such as thermal runaway, during which the rate of temperature rise is extremely large, and there is a lack of information about the large heat generation rates. In such a case, time lag between surface and core temperatures may be significant and must be accounted for.

Information about the core temperature of the cell during operation using this method can be used for a performance management as well as for taking proactive decisions for ensuring thermal safety of the cell. This work contributes towards improved thermal and electrochemical 
design of energy conversion and storage systems where overheating is a concern due to high discharge rates or unforeseen events such as thermal runaway.

\section{ACKNOWLEDGMENTS}

This material is based upon work supported by CAREER Award No. CBET-1554183 from the

National Science Foundation. Support from Office of Naval Research through grant N00014-16$1-2223$ is also gratefully acknowledged.

\section{REFERENCES}

[1] B. Scrosati, J. Hassoun, Y.-K. Sun, Lithium-ion batteries. A look into the future, Energy Environ. Sci. 4 (2011) 3287-3295. DOI: 10.1039/C1EE01388B

[2] R.A. Marsh, S. Vukson, S. Surampudi, B.V. Ratnakumar, M.C. Smart, M. Manzo, P.J. Dalton, Li ion batteries for aerospace applications, J. Power Sources 97 (2001) 25-27. DOI: 10.1016/S0378-7753(01)00584-5

[3] A. Khaligh, Z. Li, Battery, ultracapacitor, fuel cell, and hybrid energy storage systems for electric, hybrid electric, fuel cell, and plug-in hybrid electric vehicles: State of the art, IEEE Trans. Vehic. Technol. 59 (2010) 2806-2814. DOI: 10.1109/TVT.2010.2047877

[4] V. Srinivasan, C.Y. Wang, Analysis of electrochemical and thermal behavior of Li-ion cells, J. Electrochem. Soc. 150 (2003) A98-A106. DOI: 10.1149/1.1526512

[5] K.E. Thomas, J. Newman, Heats of mixing and of entropy in porous insertion electrodes, J. Power Sources 119 (2003) 844-849. DOI: 10.1016/S0378-7753(03)00283-0

[6] D. Bernardi, E. Pawlikowski, J. Newman, A general energy balance for battery systems, J. Electrochem. Soc. 132 (1985) 5-12. DOI: 10.1149/1.2113792

[7] S.J. Drake, M. Martin, D.A. Wetz, J.K. Ostanek, S.P. Miller, J.M. Heinzel, A. Jain, Heat generation rate measurement in a Li-ion cell at large C-rates through temperature and heat flux measurements, J. Power Sources 285 (2015) 266-273. DOI: 10.1016/j.jpowsour.2015.03.008

[8] G. Zhang, L. Cao, S. Ge, C.Y. Wang, C.E. Shaffer, C.D. Rahn, In situ measurement of radial temperature distributions in cylindrical li-ion cells, J. Electrochem. Soc. 161 (10) (2014) A1499A1507. DOI: 10.1149/2.0051410jes 
[9] S.J Drake, D.A. Wetz, J.K. Ostanek, S.P. Miller, J.M. Heinzel, A. Jain, Measurement of anisotropic thermophysical properties of cylindrical Li-ion cells, J. Power Sources 252 (2014) 298-304. DOI: 10.1016/j.jpowsour.2013.11.107

[10] V. Vishwakarma, C. Waghela, Z. Wei, R. Prasher, S.C. Nagpure, J. Li, F. Liu, C. Daniel, A. Jain, Heat transfer enhancement in a Lithium-ion cell through improved material-level thermal transport, J. Power Sources 300 (2015) 123-131. DOI: 10.1016/j.jpowsour.2015.09.028

[11] K. Shah, S.J. Drake, D.A. Wetz, J.K. Ostanek, S.P. Miller, J.M. Heinzel, A. Jain, An experimentally validated transient thermal model for cylindrical Li-ion cells, J. Power Sources 271 (2014) 262-268. DOI: 10.1016/j.jpowsour.2014.07.118

[12] K. Shah, S.J. Drake, D.A. Wetz, J.K. Ostanek, S.P. Miller, J.M. Heinzel, A. Jain, Modeling of steady-state convective cooling of cylindrical Li-ion cells, J. Power Sources 258 (2014) 374381. DOI: 10.1016/j.jpowsour.2014.01.115

[13] D. Anthony, D. Wong, D. Wetz, A. Jain, Improved Thermal Performance of a Li-ion Cell Through Heat Pipe Insertion, J. Electrochem. Soc., in review (2016).

[14] T.M. Bandhauer, S. Garimella, T. Fuller, A critical review of thermal issues in Lithium-ion batteries, J. Electrochem. Soc. 158 (2011) R1-R25. DOI: 10.1149/1.3515880

[15] K. Shah, V. Vishwakarma, A. Jain, Measurement of multiscale thermal transport phenomena in Li-ion cells: A review, ASME J. Electrochem. Energy Convers. \& Storage 13 (2016) 030801:1-13. DOI: $10.1115 / 1.4034413$

[16] Q. Wang, P. Ping, X. Zhao, G. Chu, J. Sun, C. Chen, Thermal runaway caused fire and explosion of lithium ion battery, J. Power Sources 208 (2012) 210-224. DOI: 10.1016/j.jpowsour.2012.02.038

[17] R. Spotnitz, J. Franklin, Abuse behavior of high-power Lithium-ion cells, J. Power Sources 113 (2003) 81-100. DOI: 10.1016/S0378-7753(02)00488-3

[18] K. Shah, D. Chalise, A. Jain, Experimental and theoretical analysis of a method to predict thermal runaway in Li-ion cells, J. Power Sources 330 (2016) 167-174. DOI: 10.1016/j.jpowsour.2016.08.133

[19] R. Zhao, S. Zhang, J. Liu, J. Gu, A review of thermal performance improving methods of lithium ion battery: Electrode modification and thermal management system, J. Power Sources 299 (2015) 557-577. DOI: 10.1016/j.jpowsour.2015.09.001

[20] N. Nieto, L. Diaz, J. Gastelurrutia, F. Blanco, J.C. Ramos, A. Rivas, Novel thermal management system design methodology for power lithium-ion battery, J. Power Sources 272 (2014) 291-302. DOI: 10.1016/j.jpowsour.2014.07.169 
[21] Z. Rao, S. Wang, M. Wu, Z. Lin, F. Li, Experimental investigation on thermal management of electric vehicle battery with heat pipe, Energy Convers. Manag. 65 (2013) 92-97. DOI: 10.1016/j.enconman.2012.08.014

[22] C.V. Hémery, F. Pra, J.F. Robin, P. Marty, Experimental performances of a battery thermal management system using a phase change material, J. Power Sources 270 (2014) 349-358. DOI: 10.1016/j.jpowsour.2014.07.147

[23] S.K. Mohammadian, Y.L. He, Y. Zhang, Internal cooling of a lithium-ion battery using electrolyte as coolant through microchannels embedded inside the electrodes, J. Power Sources 293 (2015) 458-466. DOI: 10.1016/j.jpowsour.2015.05.055

[24] S. Pannala, H. Wang, K. Kepler, S. Allu, Thermal Management for High-Capacity Large Format Li-Ion Cells, Electrochem. Soc. MA2014-02 (2014) 445.

[25] C. Forgez, D.V. Do, G. Friedrich, M. Morcrette, C. Delacourt, Thermal modeling of a cylindrical LiFePO4/graphite lithium-ion battery, J. Power Sources 195 (9) (2010) 2961-2968. DOI: 10.1016/j.jpowsour.2009.10.105

[26] Thermal Sensors: Principles and Applications for Semiconductor Industries, C.M. Jha (Ed.), Springer-Verlag, NewYork, 2015. ISBN: 978-1-4939-2580-3; DOI: 10.1007/978-1-4939-2581-0

[27] M.S.K. Mutyala, J. Zhao, J. Li, H. Pan, C. Yuan, X. Li, In-situ temperature measurement in lithium ion battery by transferable flexible thin film thermocouples, J. Power Sources 260 (2014) 43-49. DOI: 10.1016/j.jpowsour.2014.03.004

[28] R. Srinivasan, B.G. Carkhuff, M.H. Butler, A.C. Baisden, O.M. Uy, An external sensor for instantaneous measurement of the internal temperature in lithium-ion rechargeable cells, Proc. SPIE 8035, Energy Harvest. and Storage: Mater., Devices, and Appl. II (2011) 80350D. DOI: $10.1117 / 12.884691$

[29] S. Novais, M. Nascimento, L. Grande, M.F. Domingues, P. Antunes, N. Alberto, C. Leitão, R. Oliveira, S. Koch, G.T. Kim, S. Passerini, Internal and External Temperature Monitoring of a Li-Ion Battery with Fiber Bragg Grating Sensors, Sensors 16 (2016) 1394. DOI: $10.3390 / \mathrm{s} 16091394$

[30] S.J. Norton, L.R. Testardi, H.N.G. Wadley, Reconstructing Internal Temperature Distributions from Ultrasonic Time-Of-Flight Tomography and Dimensional Resonance Measurements, Proc. Ultrasonics Symp. (1983) 852-855. DOI: 10.1109/ULTSYM.1983.198180

[31] D. Anthony, D. Sarkar, A. Jain, Contactless, non-intrusive core temperature measurement of a solid body in steady-state, Int. J. Heat Mass Transfer 101 (2016) 779-788. DOI: 10.1016/j.ijheatmasstransfer.2016.05.073 
[32] D. Anthony, D. Sarkar, A. Jain, Non-invasive, transient determination of the core temperature of a heat-generating solid body, Scientific Reports 6 (2016) 35886:1-10. DOI: $10.1038 /$ srep35886

[33] Y. Ye, L.H. Saw, Y. Shi, K. Somasundaram, A.A.O. Tay, Effect of thermal contact resistances on fast charging of large format lithium ion batteries, Electrochimica Acta 134 (2014) 327-337. DOI: 10.1016/j.electacta.2014.04.134

[34] J. Zhang, B. Wu, Z. Li, J. Huang, Simultaneous estimation of thermal perameters for largeformat laminated lithium-ion batteries, J. Power Sources 259 (2014) 106-116. DOI: 10.1016/j.jpowsour.2014.02.079

[35] R.J. Moffat, 'Describing the uncertainties in experimental results,' Exp. Therm. Fluid. Sci. 1 (1988) 3-17. 


\section{List of Figures:}

Figure 1: (a) Schematic of the geometry considered in this work, (b) Picture of a 26650 Li-ion cell with embedded thermocouple. Note the black graphite film on one side of the outer surface for infrared surface temperature measurements.

Figure 2: Pictures of various steps in the fabrication of a $26650 \mathrm{Li}$-ion cell with embedded thermocouple in the core of the cell.

Figure 3: (a) Picture and (b) Schematic of the experimental setup for determining core temperature during high-rate discharge using surface temperature data acquisition and analysis.

REVISED Figure 4: Comparison of electrochemical characterization of the 26650 cell containing an embedded thermocouple and a control cell without embedded thermocouple. (a) shows a Nyquist plot, and (b) shows a voltage-capacity plot.

Figure 5: Plot of the predicted core temperature and its components $\mathrm{T}_{0, \mathrm{~A}}, \mathrm{~T}_{0, \mathrm{~B}}$ as functions of time for a 10C discharge. For reference, the core temperature measured by the embedded thermocouple is also plotted.

Figure 6: Comparison of core temperature determined from the technique described in this paper with direct measurements from the embedded thermocouple for a number of discharge rates.

Figure 7: Comparison of core temperature determined from the technique described in this paper with direct measurements from the embedded thermocouple for a discharge process where the $\mathrm{C}$ rate changes abruptly from $4 \mathrm{C}$ to $10 \mathrm{C}$.

Figure 8: Comparison of predicted core temperature with the measured mean surface temperature for 10C discharge, showing significantly higher core temperature compared to surface temperature. 


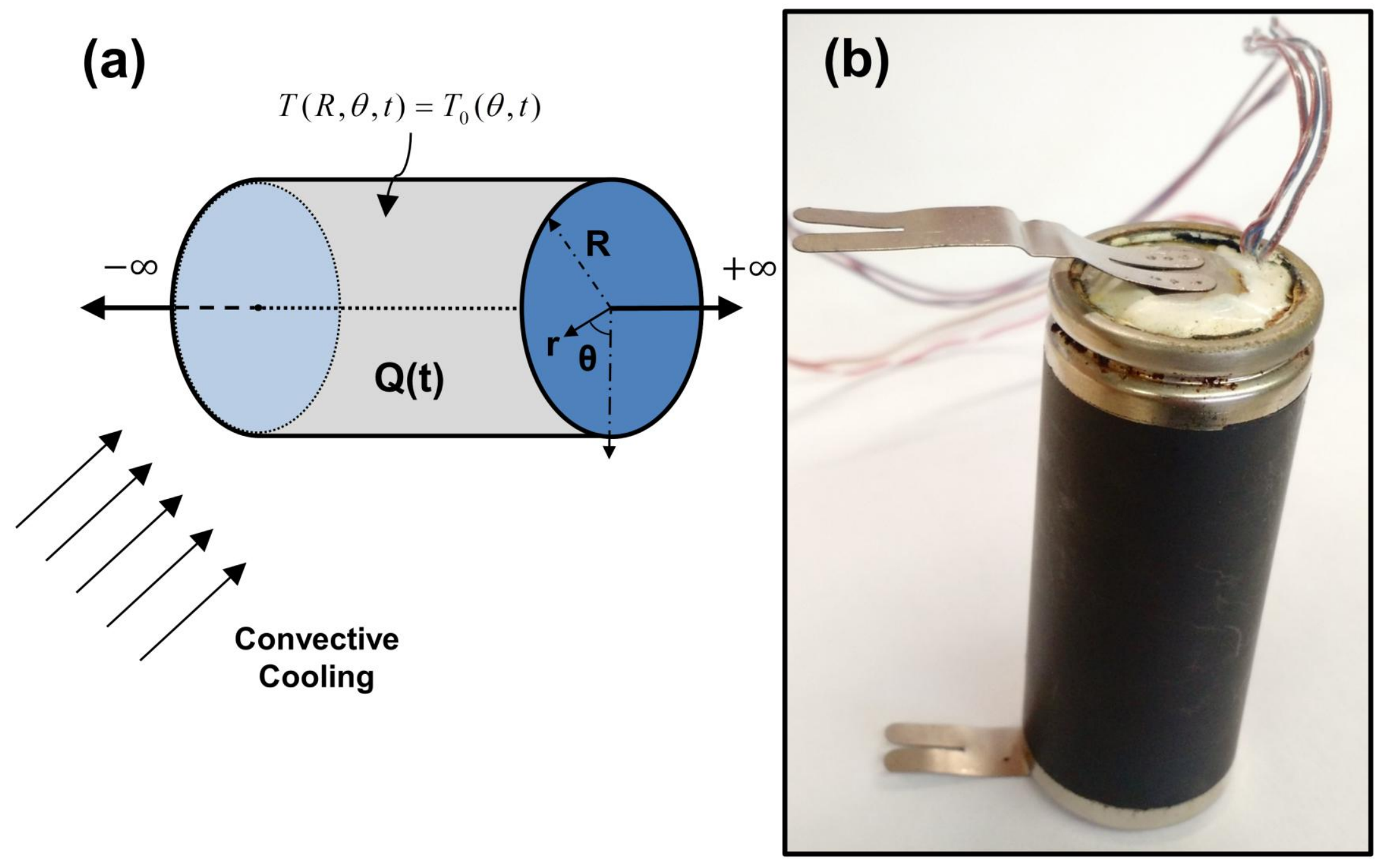

Figure 1: (a) Schematic of the geometry considered in this work, (b) Picture of a 26650 Li-ion cell with embedded thermocouple. Note the black graphite film on one side of the outer surface for infrared surface temperature measurements. 

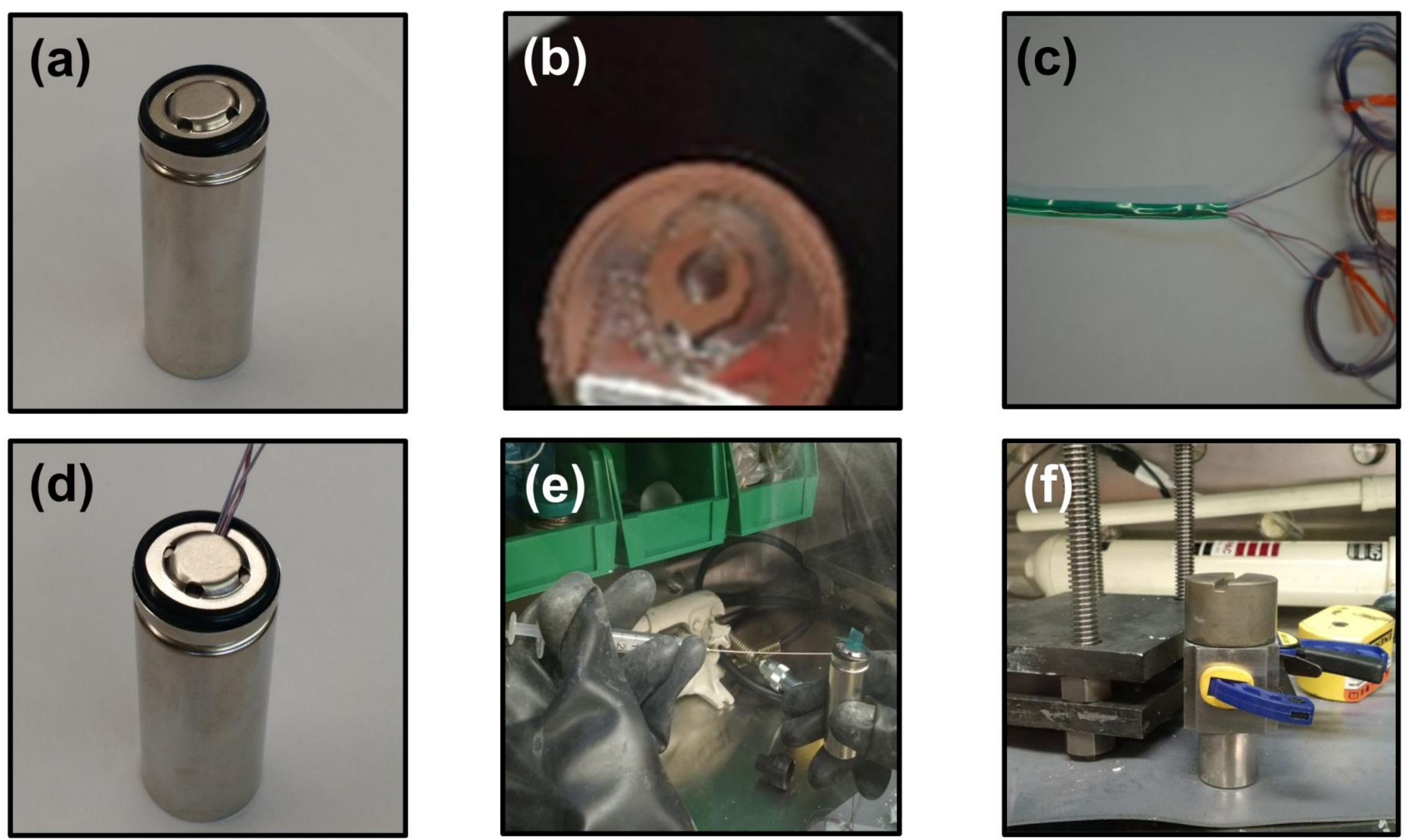

Figure 2: Pictures of various steps in the fabrication of a $26650 \mathrm{Li}$-ion cell with embedded thermocouple in the core of the cell. 
(a)

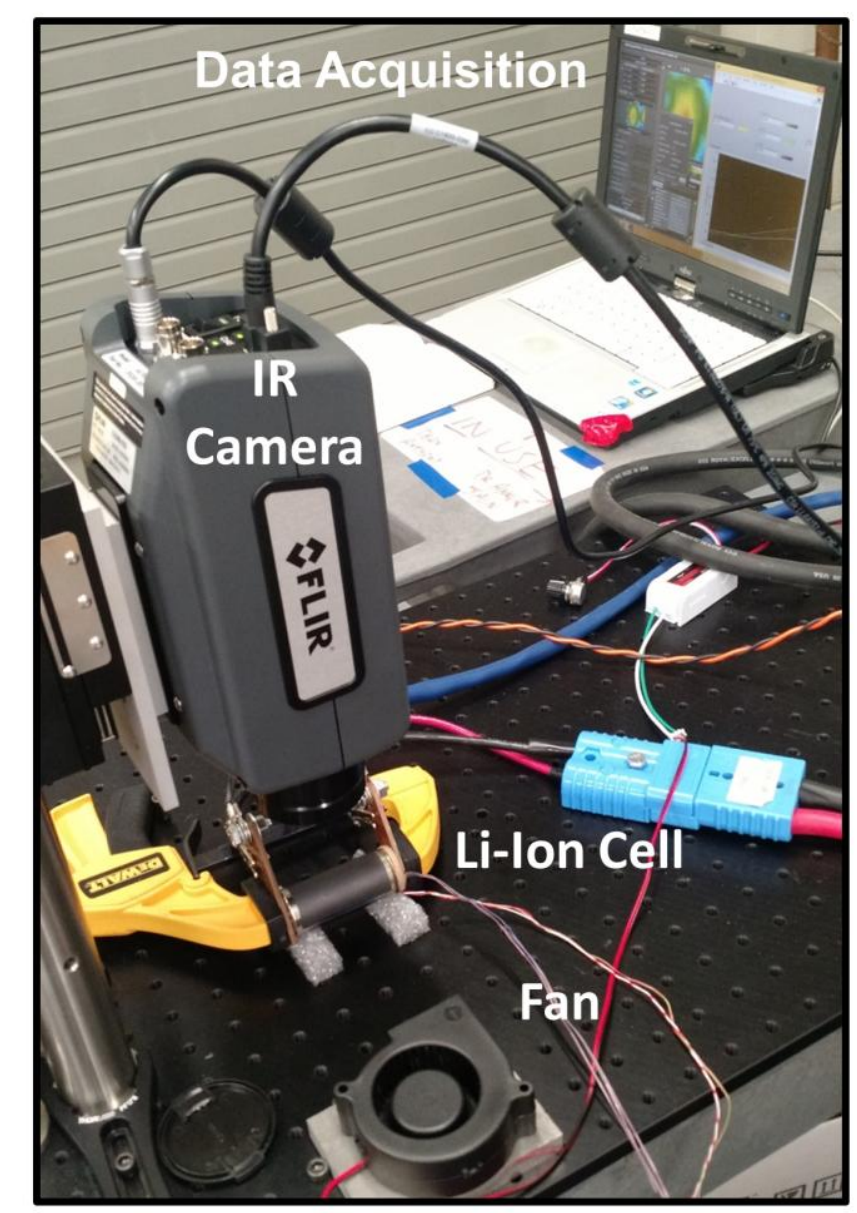

(b)

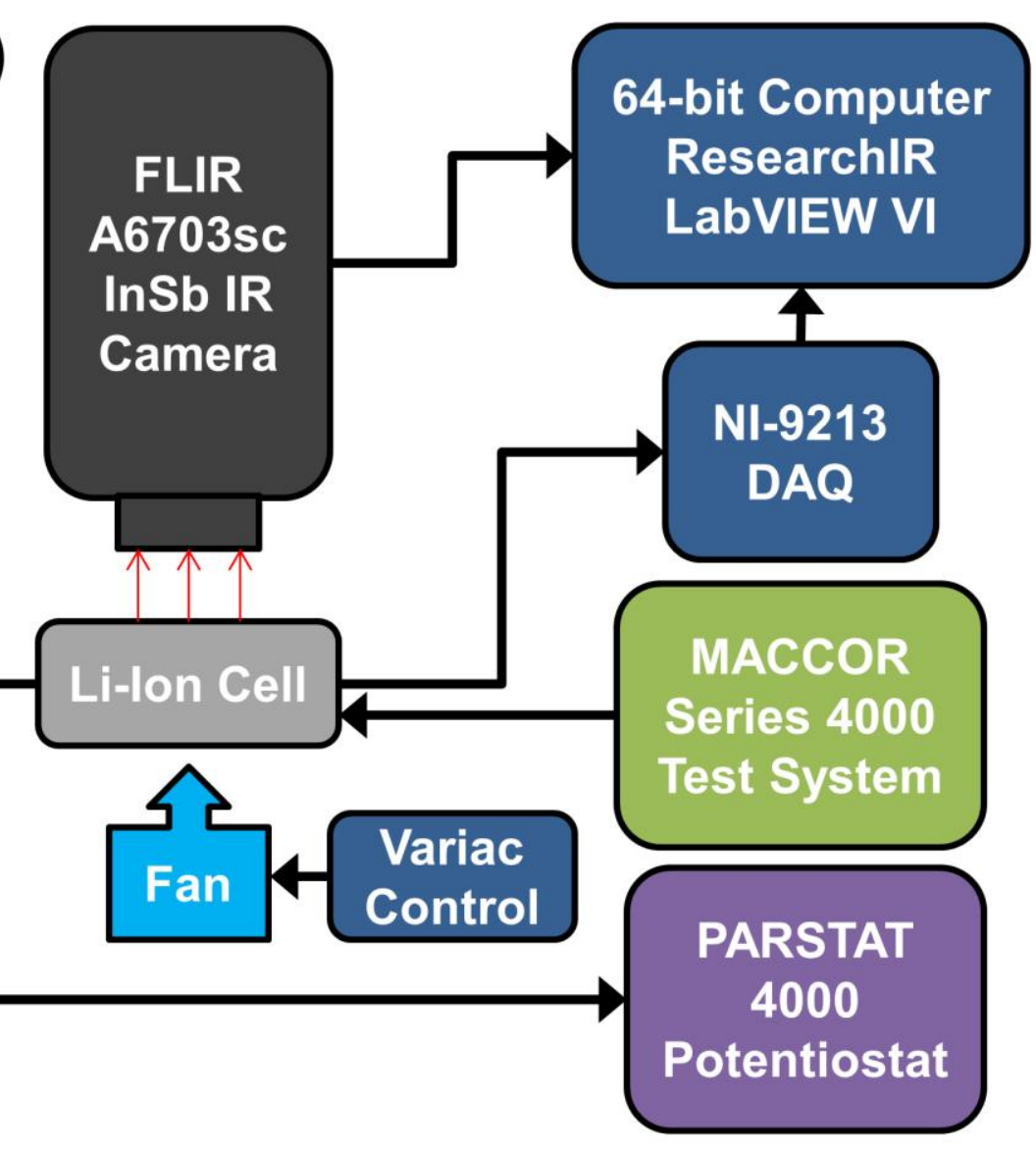

Figure 3: (a) Picture and (b) Schematic of the experimental setup for determining core temperature during high-rate discharge using surface temperature data acquisition and analysis. 

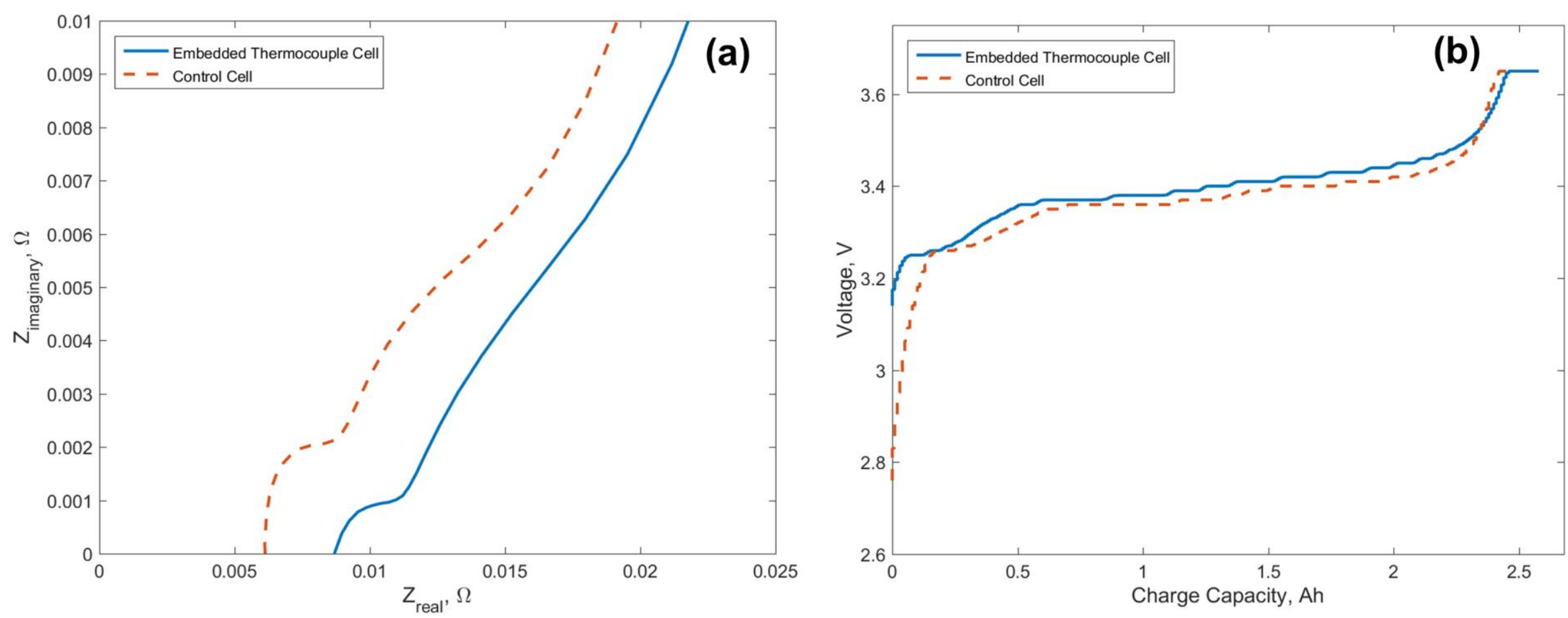

REVISED Figure 4: Comparison of electrochemical characterization of the 26650 cell containing an embedded thermocouple and a control cell without embedded thermocouple. (a) shows a Nyquist plot, and (b) shows a voltage-capacity plot. 


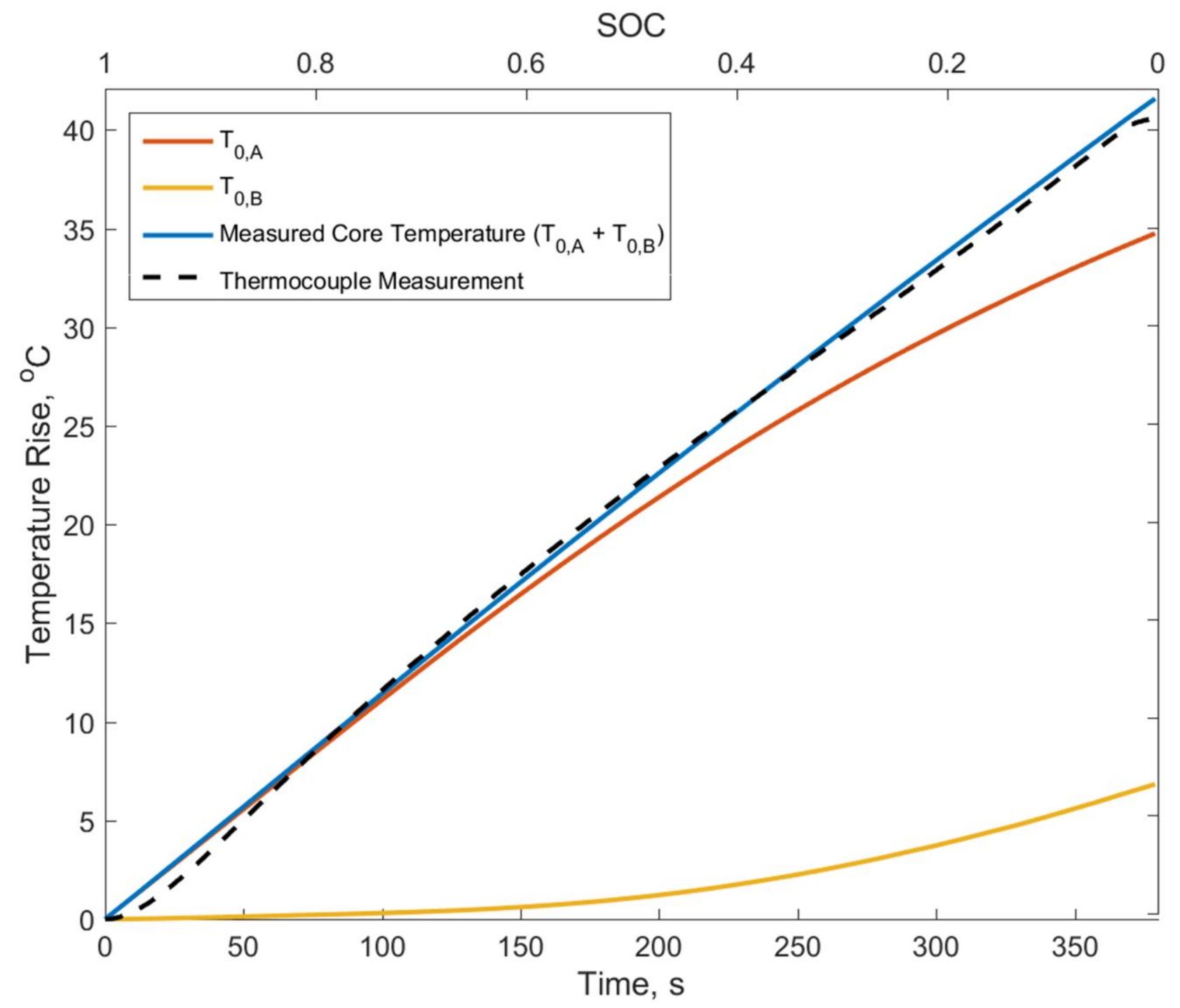

Figure 5: Plot of the predicted core temperature and its components $\mathrm{T}_{0, \mathrm{~A}}, \mathrm{~T}_{0, \mathrm{~B}}$ as functions of time for a 10C discharge. For reference, the core temperature measured by the embedded thermocouple is also plotted. 


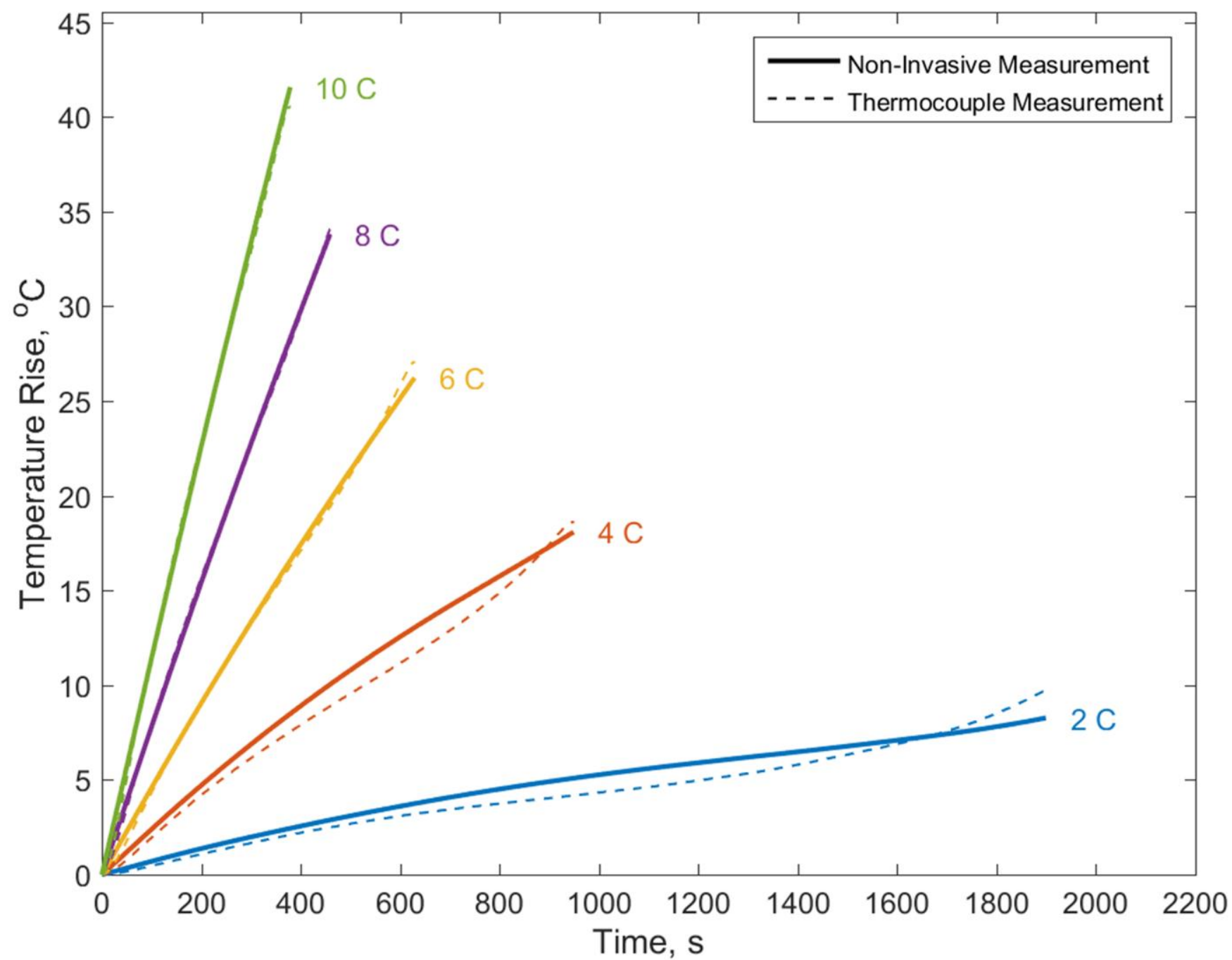

Figure 6: Comparison of core temperature determined from the technique described in this paper with direct measurements from the embedded thermocouple for a number of discharge rates. 


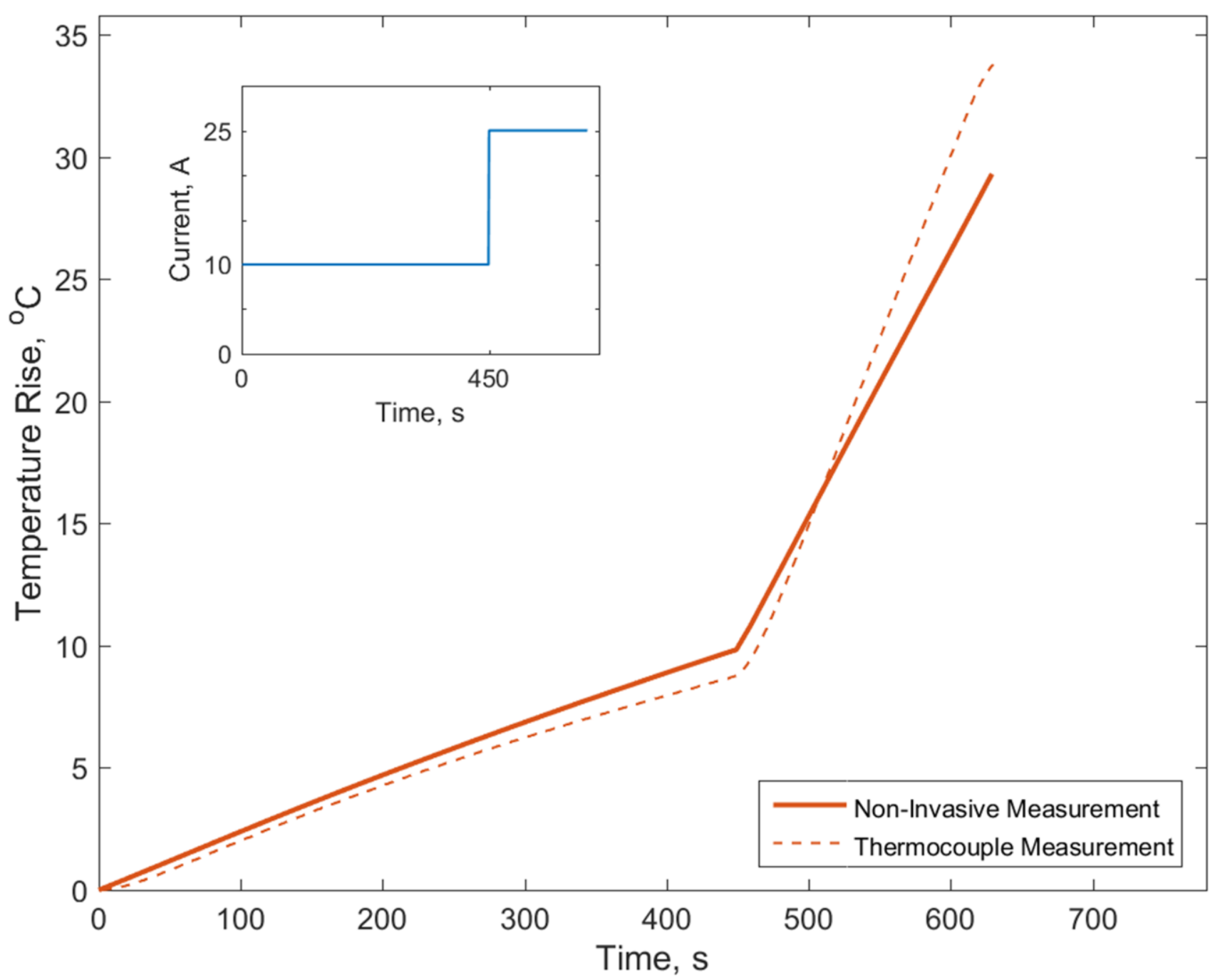

Figure 7: Comparison of core temperature determined from the technique described in this paper with direct measurements from the embedded thermocouple for a discharge process where the $\mathrm{C}$-rate changes abruptly from 4C to 10C. 


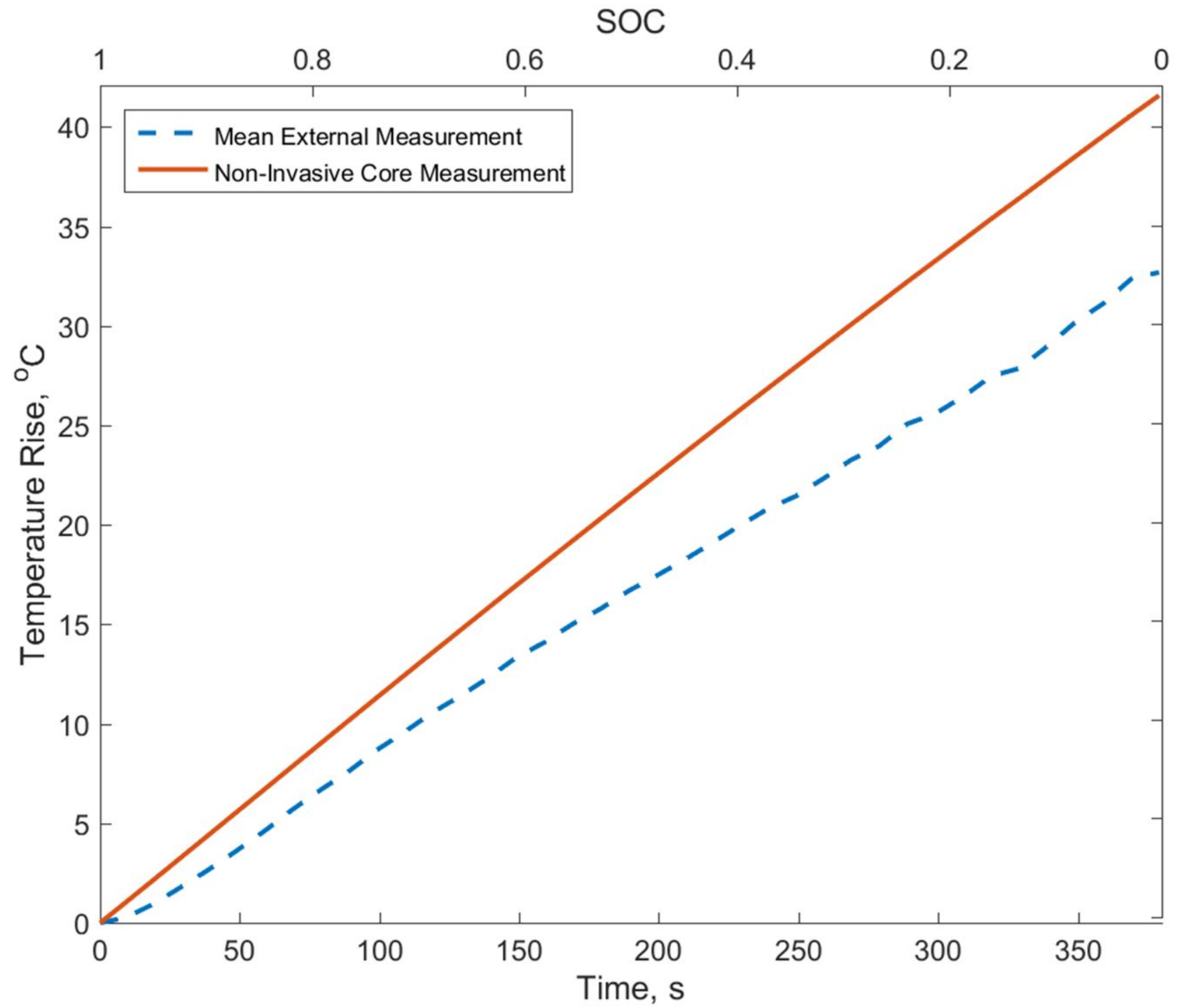

Figure 8: Comparison of predicted core temperature with the measured mean surface temperature for 10C discharge, showing significantly higher core temperature compared to surface temperature.

32 